\title{
Severity of SARS-COV-2 infection and angiotensin converting enzyme inhibitors and angiotensin receptor blockers: a meta-analysis
}

\author{
Teodoro J. Oscanoa $\odot^{1}$, Xavier Vidal $\odot^{2}$, Alfonso Carvajal $\odot^{3}$, José Amado $\odot^{1}$, \\ Roman Romero-Ortuno $\oplus^{4}$ \\ ${ }^{1}$ Facultad de Medicina, Universidad Nacional Mayor de San Marcos, Drug Safety Research Centre, Facultad de Medicina Humana, \\ Universidad de San Martín de Porres, Hospital Almenara, ESSALUD, Lima, Perú \\ ${ }^{2}$ Clinical Pharmacology Department, Vall d'Hebron Hospital, Barcelona, Spain \\ ${ }^{3}$ Centro de Estudios sobre la Seguridad de los Medicamentos (CESME), Universidad de Valladolid, Valladolid, Spain \\ ${ }^{4}$ Discipline of Medical Gerontology, Mercer's Institute for Successful Ageing, St James's Hospital, Dublin, Ireland; Global Brain Health Institute, \\ Trinity College Dublin, Ireland
}

\begin{abstract}
Background: The mechanism of entry of SARS-CoV-2 into the human host cell is through the ACE2 receptor. During the pandemic, a hypothesis has been proposed that angiotensin-converting enzyme inhibitors (ACEIs) and angiotensin II receptor blockers (ARBs) could be risk factors for the development of severe SARS-CoV-2 infection. The objective of the study was to conduct a meta-analysis of the association between ACEI or ARB use and SARS$\mathrm{CoV}-2$ infection severity or mortality.

Material and methods: We searched PubMed, EMBASE, Google scholar and the Cochrane Database of Systematic Reviews for observational studies published between December 2019 and August 4, 2020

Studies were included if they contained data on ACEI or ARB use and SARS-CoV-2 infection severity or mortality. Effect statistics were pooled using random-effects models. The quality of included studies was assessed with the Newcastle-Ottawa Scale (NOS).

Data on study design, study location, year of publication, number of participants, sex, age at baseline, outcome definition, exposure definition, effect estimates and $95 \%$ CIs were extracted.

Results: Twenty-six studies (21 cohort studies and 5 case-control studies) were identified for inclusion, combining to a total sample of 361467 participants. Mean age was 61.48 (SD 8.26) years and $51.63 \%$ were men. The mean NOS score of included studies was 7.85 (range: 7-9). Results suggested that ACEI or ARB use did not increase the risk of severe disease or mortality from SARS-CoV-2 infection (OR = 0.88, 95\% CI: 0.75-1.02, p > 0.05).

Conclusions: At present, the evidence available does not support the hypothesis of increased SARS-CoV-2 risk with ACEI or ARB drugs.
\end{abstract}

Key words: SARS-CoV-2; COVID-19; angiotensin II receptor blockers, angiotensin-converting enzyme inhibitors; renin-angiotensin system (RAS)

Address for correspondence: Teodoro J. Oscanoa, Universidad de San Martín de Porres, Facultad de Medicina Humana, Centro de Investigación de Seguridad de Medicamentos, Alameda del Corregidor 1531, La Molina 15024, Lima, Perú, Hospital Almenara ESSALUD, Lima, Perú; Universidad Nacional Mayor de San Marcos, Facultad de Medicina, Lima, Perú tel: (+511) 942 820820; e-mail: tjoscanoae@gmail.com; toscanoae@usmp.pe 


\section{Introduction}

It had not been anticipated that a phenomenon such as the appearance of the new SARS-CoV-2 pandemic would affect the world in such a short time and lead to serious health and economic consequences. Scientists around the world are in a race against time in the search for effective treatment approaches. Among the many questions that need urgent clarification is that of the possible drug-disease interactions in patients taking angiotensin-converting enzyme inhibitors (ACEIs) and angiotensin II receptor blockers (ARBs). These are among the most widely used antihypertensive drug classes in the world [1].

The renin-angiotensin system (RAS) has the function of maintaining homeostasis of blood pressure, fluid and salt in the human body. The renin-angiotensin system contains two homologous enzymes belonging to the angiotensin converting enzyme (ACE) dipeptidyl carboxypeptidase family, with different functions: the angiotensin I converting enzyme 1 (ACE1) converts angiotensin I to angiotensin II; and the angiotensin I converting enzyme 2 (ACE2) decreases the level of angiotensin II and negatively regulates the RAS system. Thus, ACE2 reduces the effects of vasoconstriction, sodium retention and fibrosis. ACE2 is expressed in various organs such as the heart, kidneys and especially in alveolar epithelial cells of the lung [2]. Under normal conditions, circulating levels of soluble ACE2 are low and its function at the lung level is minimal [3].

The mechanism of entry of SARS-CoV-2 into the human host cell is through the ACE2 receptor. SARS-CoV-2 has an envelope made up of glycoproteins, called S1 (Spike) and S2, the former binding to ACE2 on the cell surface and the latter with the cell membrane [4]. While this mechanism is similar to that of another coronavirus that caused the SARS epidemic in 2002-2003 [5, 6], SARS-CoV-2 has a higher affinity for ACE2 [7]. Although their three-dimensional structure is similar, SARS-CoV and SARS-CoV-2 differ in about $28 \%$ of the amino acid sequence in the receptor binding domains; SARS-CoV-2 has a distinct loop with flexible glycyl residues replacing rigid prolyl residues, which makes its structure less rigid and may explain its greater affinity for the ACE2 receptor [7]. Angiotensin I converting enzyme 2 was involved in the pathophysiology of SARS-CoV infection [8] and it is feared that in SARS-CoV-2 infection, this effect could be of greater magnitude. In addition, prior to the pandemic it had been reported that ACEIs/ARBs could increase mRNA expression of ACE2 at the cardiac level [9]. Hence, it has been hypothesized that ACEIs and/or ARBs could be risk factors for the development of severe forms of SARS-CoV-2 infection $[10,11]$. In the midst of the COVID-19 pandemic crisis, this simple hypothesis was, without clinical or research evidence to support it, widely disseminated by the media, which in turn caused great concern to patients who were taking these medications. Official statements rapidly followed recommending that these medications continue to be taken [12] in view of the absence of evidence to support the hypothesis. However, the absence of evidence does not mean evidence of absence and there is still an urgent need for clarification. In that light, we conducted a metaanalysis of the association between ACEI or ARB use and SARS-CoV-2 infection severity or mortality.

\section{Material and methods}

This study was conducted following the guidelines of the Preferred Reporting Items for Systematic Reviews and Meta-analyses (PRISMA) [13].

\section{Search strategy}

Two independent investigators performed a systematic search in PubMed, EMBASE, Google scholar and the Cochrane Database of Systematic Reviews for observational studies published between December 2019 and August 4, 2020. In addition, we conducted a secondary search based on the reference list of retrieved articles. The PubMed search strategy is detailed in Supplementary Table A.

\section{Eligibility criteria}

We searched for randomized controlled trials (RCTs) or observational studies reporting data on ACEI or $A R B$ use and SARS-CoV-2 infection severity or mortality. We included studies in English or other languages (all ages) meeting the following criteria: a) COVID-19 patients were diagnosed according to the interim guidance of the World Health Organization [14]; b) the study presented data on hazard ratios (HRs), relative risks (RRs), or odds ratios (ORs) with confidence intervals (CIs) or offered enough data to allow these to be calculated (including via email correspondence with original authors if necessary); and c) SARS-CoV-2 infection severity criteria were described.

\section{Quality assessment}

The quality of observational studies (cohort and case-control studies) and RCTs were assessed according to the Newcastle-Ottawa Quality Assessment Scale (NOS) [15] and the Cochrane Risk of Bias 
Assessment Tool [16], respectively. Two investigators evaluated the quality of the studies independently. Conflicting results were resolved by discussion and involvement of a third reviewer if necessary.

\section{Data extraction}

The following data were extracted from each study: authors, study location, year of publication, study design, number of participants, sex, age at baseline, outcome definition, exposure definition, effect estimates and 95\% CIs.

\section{Statistical analyses}

Primary analyses evaluated the association (HRs, RRs or ORs) between use of ACEI or ARB and SARS-CoV-2 infection severity or mortality. We used random effects with an inverse variance method to calculate the pooled RRs and 95\% CIs according to the heterogeneity between studies [17]. The overall estimates in the pooled analysis were obtained using Meta XL (www.epigear.com) add-in for Microsoft Excel.

\section{Results}

After screening 3781 citations, 26 studies (21 cohort studies and 5 case-control studies) were included (Fig. 1) [18-44], combining to a total sample of 361467participants. The characteristics of included studies are summarized in Table 1 . Thirteen studies were from China, 8 from USA and the other five being from Belgium, Italy, South Korea, Turkey and UK. Overall, mean age was 61.5 (SD 8.3) years and $51.6 \%$ were men. The mean NOS score of included studies was 7.9 (range: 7-9). The outcomes reported in the included studies are presented in Table 1.

For the meta-analysis, we used the combined outcome of severe disease and/or mortality. As shown in Figure 2, the meta-analysis suggested that ACEI or

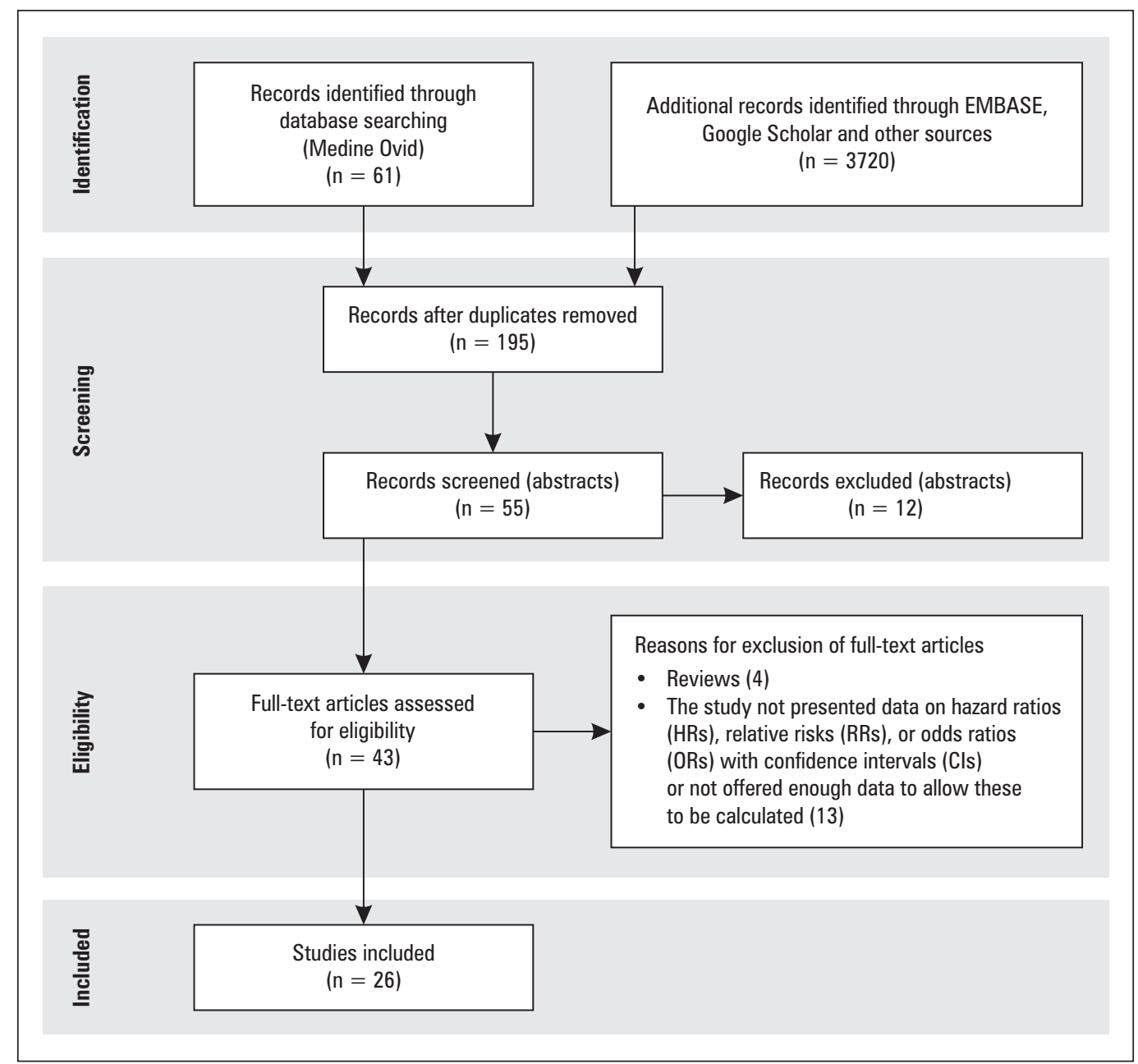

Figure 1. Flowchart of included studies 
Table 1. Characteristics of the 26 studies included in the meta-analysis

\begin{tabular}{|c|c|c|c|c|c|c|c|c|c|}
\hline Author & Country & $\begin{array}{l}\text { Study } \\
\text { design }\end{array}$ & $\begin{array}{l}\text { Total } \\
\text { sample }\end{array}$ & $\begin{array}{c}\text { Mean } \\
\text { age }\end{array}$ & $\begin{array}{c}\text { Sex } \\
\text { Male (\%) }\end{array}$ & Outcome & $\begin{array}{l}\text { Type } \\
\text { of RAS } \\
\text { inhibitor }\end{array}$ & NOS & Comorbidities \\
\hline $\begin{array}{l}\text { Guo T. et al. } \\
(2020)\end{array}$ & China & Cohort & 186 & 58.5 & 48.7 & Mortality & ACEI/ARB & 8 & $\begin{array}{c}\text { Hypertension }(33 \%) \\
\text { Coronary heart disease }(11 \%) \\
\text { Cardiomyopathy }(4 \%) \\
\text { Diabetes mellitus }(15 \%) \\
\text { COPD }(2 \%) \\
\text { Malignant neoplasm }(7 \%) \\
\text { Chronic kidney disease }(3 \%)\end{array}$ \\
\hline $\begin{array}{l}\text { Liu Y. et al. } \\
\text { (2020) }\end{array}$ & China & Cohort & 511 & 65 & 55.1 & $\begin{array}{l}\text { Severe } \\
\text { disease }\end{array}$ & $\begin{array}{l}\text { ACEI } \\
\text { ARB }\end{array}$ & 8 & Hypertension (100\%) \\
\hline $\begin{array}{l}\text { Peng Y. et al. } \\
(2020)\end{array}$ & China & Cohort & 112 & 62 & 47.32 & Mortality & ACEI/ARB & 8 & $\begin{array}{c}\text { Hypertension }(82 \%) \\
\text { Coronary heart disease }(55 \%) \\
\text { Heart failure }(36 \%) \\
\text { Diabetes mellitus }(21 \%)\end{array}$ \\
\hline $\begin{array}{l}\text { Meng J. } \\
\text { et al. (2020) }\end{array}$ & China & Cohort & 417 & 64.5 & 57.1 & $\begin{array}{l}\text { Severe } \\
\text { disease/ } \\
\text { /mortality }\end{array}$ & ACEI/ARB & 8 & Hypertension (100\%) \\
\hline $\begin{array}{l}\text { Bean D. } \\
\text { et al. }(2020)\end{array}$ & UK & Cohort & 205 & 62.95 & 51.7 & $\begin{array}{l}\text { Severe } \\
\text { disease/ } \\
\text { /mortality }\end{array}$ & $\begin{array}{l}\text { ACEI } \\
\text { ARB }\end{array}$ & 7 & $\begin{array}{c}\text { Hypertension }(51 \%) \\
\text { Diabetes mellitus }(30 \%) \text { and ischaemic } \\
\text { heart disease or heart failure }(15 \%)\end{array}$ \\
\hline $\begin{array}{l}\text { Yang G. } \\
\text { et al. (2020) }\end{array}$ & China & Cohort & 462 & 67 & 49.4 & $\begin{array}{l}\text { Severe } \\
\text { disease/ } \\
\text { /mortality }\end{array}$ & ACEI/ARB & 9 & $\begin{array}{c}\text { Hypertension }(100 \%) \\
\text { Diabetes mellitus }(30 \%) \\
\text { Respiratory disease, cardiopathy }(18 \%) \\
\text { Neurological disease }(8 \%)\end{array}$ \\
\hline $\begin{array}{l}\text { Feng Y. et al. } \\
(2020)\end{array}$ & China & Cohort & 476 & 53 & 56.9 & $\begin{array}{l}\text { Severe } \\
\text { disease }\end{array}$ & $\begin{array}{c}\text { ACEl/ARB } \\
\text { ARB } \\
\text { ACEI }\end{array}$ & 8 & $\begin{array}{c}\text { Hypertension (24\%) } \\
\text { Cardiovascular disease (8\%) } \\
\text { Diabetes mellitus (10\%) } \\
\text { Malignancy (3\%) } \\
\text { Cerebrovascular disease (4\%) } \\
\text { Immunosuppression (2\%) } \\
\text { COPD (5\%) }\end{array}$ \\
\hline $\begin{array}{l}\text { Feng } Z \text {. } \\
\text { et al. (2020) }\end{array}$ & China & Cohort & 564 & 47 & 50.4 & $\begin{array}{l}\text { Severe } \\
\text { disease }\end{array}$ & ACEI/ARB & 8 & $\begin{array}{c}\text { Hypertension (15\%) } \\
\text { Diabetes mellitus }(8 \%) \\
\text { Cardiovascular disease (4\%) } \\
\text { COPD (3\%) } \\
\text { Hepatitis B/C infection (2\%) } \\
\text { Cerebrovascular disease (1\%) }\end{array}$ \\
\hline $\begin{array}{l}\text { Rentsch C. } \\
\text { et al. (2020) }\end{array}$ & USA & Cohort & 585 & 66.1 & 95.4 & $\begin{array}{l}\text { Severe } \\
\text { disease }\end{array}$ & $\begin{array}{c}\text { ACEI/ARB } \\
\text { ARB } \\
\text { ACEI }\end{array}$ & 7 & $\begin{array}{c}\text { Asthma (8\%) } \\
\text { Cancer (15\%) } \\
\text { Chronic kidney disease (15\%) } \\
\text { COPD (26\%) } \\
\text { Diabetes mellitus }(33 \%) \\
\text { Hypertension }(65 \%) \\
\text { Liver disease }(12 \%) \\
\text { Vascular disease }(29 \%)\end{array}$ \\
\hline $\begin{array}{l}\text { Zeng Z. } \\
\text { et al. (2020) }\end{array}$ & China & Cohort & 274 & 60 & 45 & Mortality & ACEI/ARB & 8 & $\begin{array}{c}\text { Hypertension (100\%) } \\
\text { COPD (6\%) } \\
\text { Chronic renal insufficiency (2\%) } \\
\text { Cardiovascular disease }(11 \%) \\
\text { Diabetes mellitus (15\%) } \\
\text { Cerebrovascular disease }(8 \%)\end{array}$ \\
\hline $\begin{array}{l}\text { Zhang P. } \\
\text { et al. }(2020)\end{array}$ & China & Cohort & 1128 & 64 & 53.2 & Mortality & ACEI/ARB & 7 & $\begin{array}{c}\text { Hypertension }(100 \%) \\
\text { Diabetes mellitus }(23 \%) \\
\text { Coronary heart disease }(15 \%) \\
\text { Chronic renal diseases }(4 \%) \\
\text { Cerebrovascular diseases }(3 \%)\end{array}$ \\
\hline
\end{tabular}


Table 1. Characteristics of the 26 studies included in the meta-analysis

\begin{tabular}{|c|c|c|c|c|c|c|c|c|c|}
\hline Author & Country & $\begin{array}{l}\text { Study } \\
\text { design }\end{array}$ & $\begin{array}{l}\text { Total } \\
\text { sample }\end{array}$ & $\begin{array}{c}\text { Mean } \\
\text { age }\end{array}$ & $\begin{array}{c}\text { Sex } \\
\text { Male (\%) }\end{array}$ & Outcome & $\begin{array}{l}\text { Type } \\
\text { of RAS } \\
\text { inhibitor }\end{array}$ & NOS & Comorbidities \\
\hline $\begin{array}{l}\text { Li J. et al. } \\
\text { (2020) }\end{array}$ & China & Cohort & 1178 & 55.5 & 46.3 & Mortality & $\begin{array}{c}\text { ACEI/ARB } \\
\text { ARB } \\
\text { ACEI }\end{array}$ & 9 & $\begin{array}{c}\text { Hypertension }(100 \%) \\
\text { Cerebrovascular disease }(19 \%) \\
\text { Coronary heart disease }(17 \%) \\
\text { Heart failure }(3 \%) \\
\text { Diabetes mellitus }(35 \%) \\
\text { Digestive disorder }(22 \%)\end{array}$ \\
\hline $\begin{array}{l}\text { Choi H. et al. } \\
(2020)\end{array}$ & $\begin{array}{l}\text { South } \\
\text { Korea }\end{array}$ & CC & 1585 & 63 & 42.7 & $\begin{array}{l}\text { Severe } \\
\text { disease/ } \\
\text { /mortality }\end{array}$ & ACEI/ARB & 8 & $\begin{array}{c}\text { Hypertension (100\%) } \\
\text { Diabetes mellitus }(47 \%) \\
\text { Major neurologic diseases (28\%) } \\
\text { Chronic lung diseases (19\%) }\end{array}$ \\
\hline $\begin{array}{l}\text { Hu J. et al. } \\
(2020)\end{array}$ & China & CC & 149 & 57 & 59.06 & $\begin{array}{l}\text { Severe } \\
\text { disease }\end{array}$ & ACEI/ARB & 7 & $\begin{array}{c}\text { Diabetes mellitus }(20 \%) \\
\text { Heart disease }(5 \%) \\
\text { COPD (1\%) } \\
\text { Chronic liver disease }(6 \%) \\
\text { Chronic renal disease }(4 \%) \\
\text { Cancer }(2 \%)\end{array}$ \\
\hline $\begin{array}{l}\text { Zhou X. } \\
\text { et al. (2020) }\end{array}$ & China & CC & 110 & 57.7 & 54.5 & Mortality & ACEI/ARB & 7 & $\begin{array}{c}\text { Hypertension }(33 \%) \text { and diabetes } \\
\text { mellitus (10\%) } \\
\text { Cardiovascular disease (9\%) } \\
\text { Chronic liver disease (4\%) } \\
\text { Malignancy }(4 \%)\end{array}$ \\
\hline $\begin{array}{l}\text { Huang Z. } \\
\text { et al. }(2020)\end{array}$ & China & Cohort & 50 & 52.65 & 54 & $\begin{array}{l}\text { Severe } \\
\text { disease/ } \\
\text { /mortality }\end{array}$ & ACEI/ARB & 8 & $\begin{array}{c}\text { Diabetes mellitus }(13 \%) \\
\text { Coronary artery disease }(3 \%) \\
\text { COPD }(5 \%) \\
\text { Chronic obstructive pulmonary disease } \\
(5 \%)\end{array}$ \\
\hline $\begin{array}{l}\text { De Spiege- } \\
\text { leer A. et al. } \\
(2020)\end{array}$ & Belgium & Cohort & 154 & 86 & 33.1 & $\begin{array}{l}\text { Severe } \\
\text { disease }\end{array}$ & ACEI/ARB & 8 & $\begin{array}{c}\text { Hypertension (25\%) } \\
\text { Diabetes (18\%) }\end{array}$ \\
\hline $\begin{array}{l}\text { Ip A. et al. } \\
(2020)\end{array}$ & USA & Cohort & 1129 & $\begin{array}{l}\text { Miss- } \\
\text { ing }\end{array}$ & Missing & Mortality & $\begin{array}{c}\text { ACEI/ARB } \\
\text { ARB } \\
\text { ACEI }\end{array}$ & 7 & Hypertension (100\%) \\
\hline $\begin{array}{l}\text { Khera R. } \\
\text { et al. }(2020)\end{array}$ & USA & Cohort & 10196 & 69 & 45.4 & Mortality & $\begin{array}{l}\text { ACEI } \\
\text { ARB }\end{array}$ & 8 & $\begin{array}{c}\text { Hypertension }(100 \%) \\
\text { Diabetes without complications (51\%) } \\
\text { Myocardial infarction (5\%) } \\
\text { Chronic heart failure }(31 \%) \\
\text { Chronic pulmonary disease }(39 \%)\end{array}$ \\
\hline $\begin{array}{l}\text { Reynolds H. } \\
\text { et al. (2020) }\end{array}$ & USA & Cohort & 12594 & 49 & 41.5 & $\begin{array}{l}\text { Severe } \\
\text { disease }\end{array}$ & $\begin{array}{c}\text { ACEI/ARB } \\
\text { ARB } \\
\text { ACEI }\end{array}$ & 9 & $\begin{array}{c}\text { Hypertension (35\%) } \\
\text { Heart failure (6\%) } \\
\text { Myocardial infarction (4\%) } \\
\text { Diabetes (18\%) } \\
\text { Chronic kidney disease }(10 \%) \\
\text { Obstructive lung disease }(15 \%)\end{array}$ \\
\hline $\begin{array}{l}\text { Richardson S. } \\
\text { et al. }(2020)\end{array}$ & USA & Cohort & 5700 & 63 & 60.3 & Mortality & ACEI/ARB & 9 & $\begin{array}{c}\text { Hypertension }(57 \%) \\
\text { Obesity (42\%) } \\
\text { Diabetes mellitus (32\%) }\end{array}$ \\
\hline $\begin{array}{l}\text { Chaudhri l. } \\
\text { et al. (2020) }\end{array}$ & USA & Cohort & 300 & 59.1 & 60 & $\begin{array}{l}\text { Severe } \\
\text { disease }\end{array}$ & ACEI/ARB & 7 & $\begin{array}{c}\text { Hypertension }(44 \%) \\
\text { Diabetes mellitus }(25 \%) \text { and/or heart } \\
\text { failure }(15 \%)\end{array}$ \\
\hline $\begin{array}{l}\text { Dublin S. } \\
\text { et al. (2020) }\end{array}$ & USA & Cohort & 322044 & 51 & 46 & $\begin{array}{l}\text { Severe } \\
\text { disease }\end{array}$ & ACEI/ARB & 7 & \\
\hline
\end{tabular}


Table 1. Characteristics of the 26 studies included in the meta-analysis

\begin{tabular}{|c|c|c|c|c|c|c|c|c|c|}
\hline Author & Country & $\begin{array}{l}\text { Study } \\
\text { design }\end{array}$ & $\begin{array}{c}\text { Total } \\
\text { sample }\end{array}$ & $\begin{array}{c}\text { Mean } \\
\text { age }\end{array}$ & $\begin{array}{c}\text { Sex } \\
\text { Male (\%) }\end{array}$ & Outcome & $\begin{array}{l}\text { Type } \\
\text { of RAS } \\
\text { inhibitor }\end{array}$ & NOS & Comorbidities \\
\hline $\begin{array}{l}\text { Felice et al. } \\
(2020)\end{array}$ & Italy & CC & 133 & 73.1 & 28 & Mortality & $\begin{array}{c}\text { ACEI/ARB } \\
\text { ARB } \\
\text { ACEI }\end{array}$ & 8 & $\begin{array}{c}\text { Hypertension (100\%) } \\
\text { Chronic heart failure (18\%) } \\
\text { Diabetes mellitus (26\%) } \\
\text { Cancer (16\%) } \\
\text { Chronic obstructive pulmonary } \\
\text { disease }(11 \%)\end{array}$ \\
\hline $\begin{array}{l}\text { Lam K. el al. } \\
(2020)\end{array}$ & USA & CC & 614 & 68 & 56.4 & Mortality & ACEI/ARB & 8 & $\begin{array}{c}\text { Hypertension }(100 \%) \\
\text { Diabetes }(41 \%) \\
\text { Asthma }(5 \%) \\
\text { Coronary heart disease }(24 \%) \\
\text { COPD }(13 \%) \\
\text { Heart failure }(13 \%) \\
\text { Cancer }(12 \%) \\
\text { Chronic kidney disease }(16 \%)\end{array}$ \\
\hline $\begin{array}{l}\text { Senkal N. } \\
\text { et al. (2020) }\end{array}$ & Turkey & Cohort & 611 & 63 & 53.2 & $\begin{array}{l}\text { Severe } \\
\text { disease }\end{array}$ & $\begin{array}{l}\text { ACEI } \\
\text { ARB }\end{array}$ & 8 & $\begin{array}{c}\text { Diabetes mellitus (41\%) } \\
\text { COPD/asthma ( } 14 \%) \\
\text { Coronary artery disease history (26\%) } \\
\text { Congestive heart failure }(9 \%)\end{array}$ \\
\hline
\end{tabular}

ACEI — angiotensin-converting enzyme inhibitor; ARB — angiotensin II receptor blocker; SD — standard deviation; Cl — confidence interval; RAS — renin-angiotensin system; NOS — Newcastle-Ottawa Scale; $\mathrm{CC}$ - case control; COPD — chronic obstructive pulmonary disease

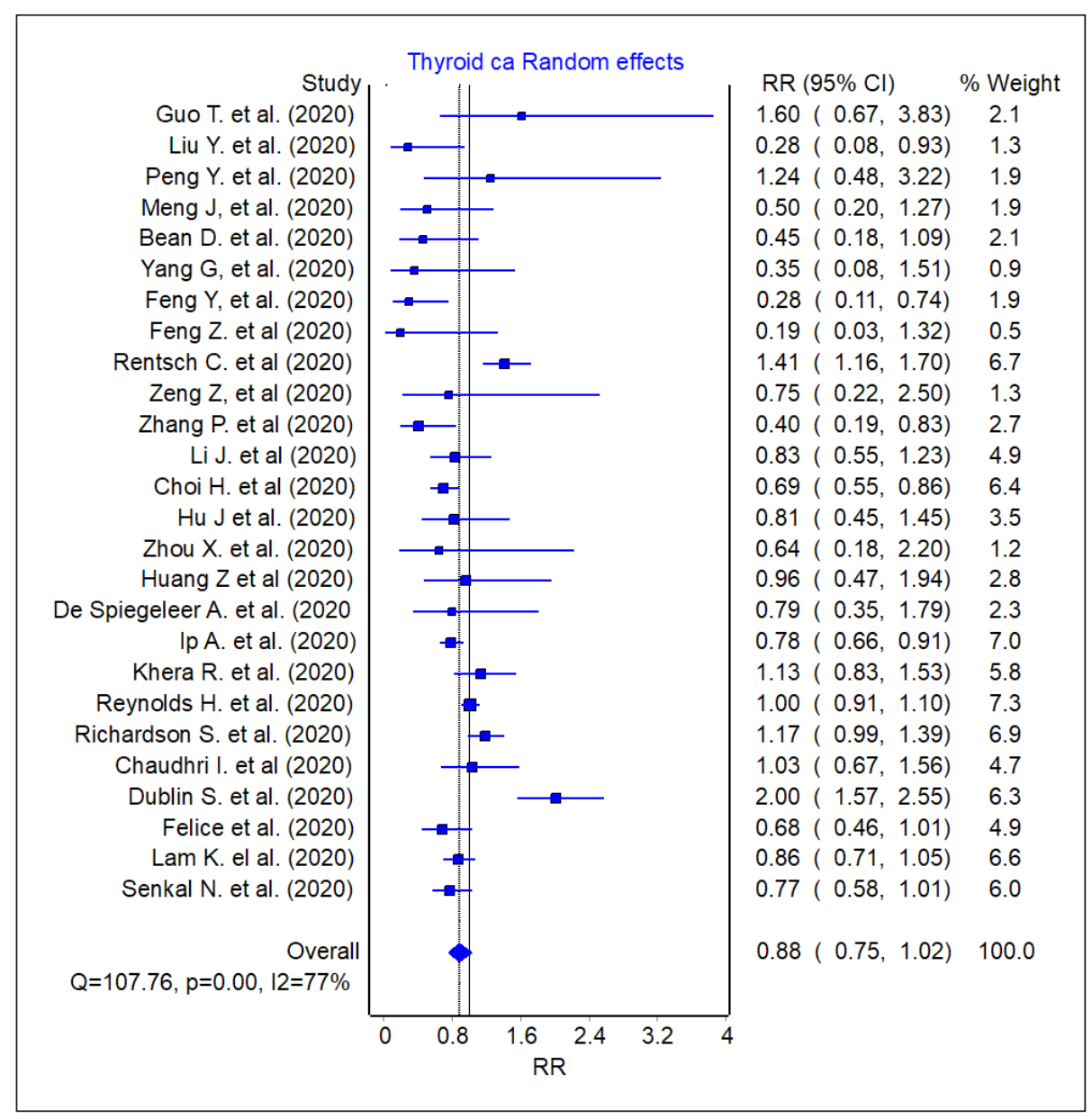

Figure 2. Forest plot of the meta-analysis of the association between angiotensin-converting enzyme inhibitor (ACEI) or angiotensin II receptor blocker (ARB) use and SARS-CoV-2 infection severity or mortality. Analysis model: random effect. OR — odds ratio; $\mathrm{Cl}$ — confidence interval 
Table 2. Association between the use of angiotensin-converting enzyme inhibitor (ACEI) or angiotensin II receptor blocker (ARB) and SARS-CoV-2 infection severity or mortality: summary of subgroup analyses

\begin{tabular}{|l|c|c|c|}
\hline Subgroup & Studies (n) & $\begin{array}{c}\text { Adjusted OR } \\
(\mathbf{9 5 \%} \text { CI) }\end{array}$ & $\mathbf{p}$ \\
\hline $\begin{array}{l}\text { Mean age (years) } \\
\quad<60\end{array}$ & 10 & $0.96(0.71-1.30)$ & $>0.05$ \\
$\geq 60$ & 16 & $0.82(0.67-1.0)$ & $>0.05$ \\
\hline Male sex (\%) & 18 & $0.84(0.67-1.04)$ & $>0.05$ \\
$\quad<55$ & 8 & $0.88(0.69-1.12)$ & $>0.05$ \\
$\geq 55$ & 12 & $0.90(0.76-1.06)$ & $>0.05$ \\
\hline Type of RAS inhibitors & 11 & $0.90(0.74-1.08)$ & $>0.05$ \\
ARB & & & \\
ACEI & 12 & \\
\hline
\end{tabular}

$\mathrm{RAS}$ - renin-angiotensin system; $\mathrm{OR}$ — odds ratio; $\mathrm{Cl}$ — confidence interval

ARB use did not increase the risk of severe disease or mortality from SARS-CoV-2 infection $(\mathrm{OR}=0.88$, 95\% CI: 0.75-1.02, p > 0.05). Subgroup analyses were conducted to assess ACEI and ARB effects separately (Tab. 2) but no significant associations were found. Subgroup analyses were also negative for the effects of age ( $<60$ vs. $60+)$ or sex (Tab. 2).

\section{Discussion}

Our study found no evidence to support the hypothesis of increased SARS-CoV-2 risk with ACEI or ARB drugs. This would seem at odds with a previous finding that chronic use of ACEIs and ARBs was high among intensive care unit patients with non-COVID-19 sepsis [45]. However, it is possible that ACEIs/ARBs could be a marker of underlying comorbidities rather than being causal in SARS-CoV-2 severity or mortality.

Prior to the SARS-CoV-2 pandemic, Shinohara et al. published a meta-analytic study where they found a decreased risk of post-stroke pneumonia in patients treated with ACEIs compared to other antihypertensive drugs (RR: 0.61, 95\% CI: 0.51-0.75; $\mathrm{p}<0.001)$ [46]. In another meta-analysis, Liu et al. found that ARBs were associated with a decreased risk of pneumonia morbidity $(\mathrm{OR}=0.55,95 \% \mathrm{CI}$ : $0.43-0.70, \mathrm{p}<0.01)$ and mortality $(\mathrm{OR}=0.55$, 95\% CI: $0.44-0.69, \mathrm{p}<0.01$ ) [19]. These findings could however be seen from the perspective that the prescription of ACEIs/ARBs may be a marker of good general medical care, given the well-evidenced preventative role of these medications in many cardiovascular and metabolic diseases.

The basis for the hypothesis of a probable ACEI/ /ARB-induced increase in ACE2 expression has been recently revised, evaluating the results of 12 animal and 11 human studies [47]. In animal studies, no significant changes in ACE2 expression were found, and in those where it was evidenced, it was when models of acute injury were used or at higher doses than those used in humans; furthermore, no increase in ACE2 expression induced by ACEIs/ARBs was evidenced in human studies [47].

It has been proposed that ARBs may have protective effects on severity and mortality in SARS$\mathrm{CoV}-2$ infection through increasing the production of angiotensin 1-7, reducing angiotensin II and contributing towards lung protection [48]. Recently, Liu et al. found that angiotensin II levels in the plasma of COVID-19 infected patients was markedly elevated and linearly associated to viral load and lung injury [49]. However, this may be a marker of general physiological stress during severe acute illness and not have specific drug-disease implications.

Our study is limited in that it only relies on observational studies and not RCTs and includes a relatively small number of participants. It would be important that future, more powered studies, re-evaluate the possible relevance of age (young vs. old), sex, and possible different roles of ACEI and ARB drugs. It would also be important to assess the risk of specific comorbidities (e.g. diabetes, hypertension, cerebrovascular disease, ischaemic heart disease) in the absence of ACEI/ARB drugs. For example, Feng et al. showed that hypertension without ACEI/ARB therapy was an independent risk factor for developing severe pneumonia irrespective of age [42].

In conclusion, the evidence available at present does not support the hypothesis of increased SARS$\mathrm{CoV}-2$ risk with ACEI or ARB drugs. However, more evidence needs to accumulate before this controversy can be resolved; in the meantime, clinicians may adopt a tailored, pragmatic approach that is supported by official recommendations $[12,50]$. 


\section{Funding}

None.

\section{Conflict of interest}

None.

\section{References}

1. Derington CG, King JB, Herrick JS, et al. Trends in Antihypertensive Medication Monotherapy and Combination Use Among US Adults, National Health and Nutrition Examination Survey 2005-2016. Hypertension. 2020; 75(4): 973-981, doi: 10.1161/HYPERTENSIONAHA.119.14360, indexed in Pubmed: 32148129.

2. Hamming I, Timens W, Bulthuis MLC, et al. Tissue distribution of ACE2 protein, the functional receptor for SARS coronavirus. A first step in understanding SARS pathogenesis. J Pathol. 2004; 203(2): 631-637, doi: 10.1002/path.1570, indexed in Pubmed: 15141377.

3. Serfozo P, Wysocki J, Gulua G, et al. Ang II (Angiotensin II) Conversion to Angiotensin-(1-7) in the Circulation Is POP (Prolyloligopeptidase)-Dependent and ACE2 (AngiotensinConverting Enzyme 2)-Independent. Hypertension. 2020; 75(1): 173-182, doi: 10.1161/HYPERTENSIONAHA.119.14071, indexed in Pubmed: 31786979.

4. Wahba L, Jain N, Fire AZ, et al. A pneumonia outbreak associated with a new coronavirus of probable bat origin. Nature. 2020; 579(7798): 270-273, doi: 10.1038/s41586-020-2012-7, indexed in Pubmed: 32015507.

5. Ge XY, Li JL, Yang XL, et al. Isolation and characterization of a bat SARS-like coronavirus that uses the ACE2 receptor. Nature. 2013; 503(7477): 535-538, doi: 10.1038/nature12711, indexed in Pubmed: 24172901.

6. Prabakaran P, Xiao X, Dimitrov DS. A model of the ACE2 structure and function as a SARS-CoV receptor. Biochem Biophys Res Commun. 2004; 314(1): 235-241, doi: 10.1016/j.bbrc.2003.12.081, indexed in Pubmed: 14715271.

7. Cheng ZJ, Shan J. 2019 Novel coronavirus: where we are and what we know. Infection. 2020; 48(2): 155-163, doi: 10.1007/s15010020-01401-y, indexed in Pubmed: 32072569.

8. de Wit E, van Doremalen N, Falzarano D, et al. SARS and MERS: recent insights into emerging coronaviruses. Nat Rev Microbiol. 2016; 14(8): 523-534, doi: 10.1038/nrmicro.2016.81, indexed in Pubmed: 27344959.

9. Ferrario CM, Jessup J, Chappell MC, et al. Effect of angiotensin-converting enzyme inhibition and angiotensin II receptor blockers on cardiac angiotensin-converting enzyme 2. Circulation. 2005; 111(20): 2605-2610, doi: 10.1161/CIRCULATIONAHA.104.510461, indexed in Pubmed: 15897343.

10. Sommerstein R, Kochen MM, Messerli FH, et al. Coronavirus Disease 2019 (COVID-19): Do Angiotensin-Converting Enzyme Inhibitors/Angiotensin Receptor Blockers Have a Biphasic Effect? J Am Heart Assoc. 2020; 9(7): e016509, doi: 10.1161/ JAHA.120.016509, indexed in Pubmed: 32233753.

11. Fang L, Karakiulakis G, Roth M. Are patients with hypertension and diabetes mellitus at increased risk for COVID-19 infection? Lancet Respir Med. 2020; 8(4): e21, doi: 10.1016/s2213-2600(20)301168, indexed in Pubmed: 32171062.

12. Bavishi C, Maddox TM, Messerli FH. Coronavirus Disease 2019 (COVID-19) Infection and Renin Angiotensin System Blockers. JAMA Cardiol. 2020 [Epub ahead of print], doi: 10.1001/jamacardio.2020.1282, indexed in Pubmed: 32242890.

13. Moher D, Liberati A, Tetzlaff J, et al. Preferred Reporting Items for Systematic Reviews and Meta-Analyses: The PRISMA Statement. PLoS Med. 2009; 6: e1000097, doi: 10.1371/journal. pmed.1000097, indexed in Pubmed: 19621072.

14. World Health Organization. Clinical management of severe acute respiratory infection when novel coronavirus (2019-nCoV) infection is suspected: interim guidance, 28 January 2020. https://apps.who. int/iris/handle/10665/330893.
15. Stang A. Critical evaluation of the Newcastle-Ottawa scale for the assessment of the quality of nonrandomized studies in meta-analyses. Eur J Epidemiol. 2010; 25(9): 603-605, doi: 10.1007/s10654-0109491-z, indexed in Pubmed: 20652370.

16. Cumpston M, Li T, Page MJ, et al. Updated guidance for trusted systematic reviews: a new edition of the Cochrane Handbook for Systematic Reviews of Interventions. Cochrane Database Syst Rev. 2019; 10: ED000142, doi: 10.1002/14651858.ED000142, indexed in Pubmed: 31643080.

17. DerSimonian R, Laird N. Meta-analysis in clinical trials. Contemp Clin Trials. 1986; 7(3): 177-188, doi: 10.1016/01972456(86)90046-2, indexed in Pubmed: 26343745.

18. Guo T, Fan Y, Chen M, et al. Cardiovascular Implications of Fatal Outcomes of Patients With Coronavirus Disease 2019 (COVID-19). JAMA Cardiol. 2020 [Epub ahead of print], doi: 10.1001/jamacardio.2020.1017, indexed in Pubmed: 32219356.

19. Liu Y, Huang F, Xu J, et al. Anti-hypertensive Angiotensin II receptor blockers associated to mitigation of disease severity in elderly COVID-19 patients. medRxiv (preprint). , doi: 10.1101/2020.0 3.20.20039586

20. Zhang P, Zhu L, Cai J, et al. Association of Inpatient Use of Angiotensin-Converting Enzyme Inhibitors and Angiotensin II Receptor Blockers With Mortality Among Patients With Hypertension Hospitalized With COVID-19. Circ Res. 2020; 126(12): 1671-1681, doi: 10.1161/CIRCRESAHA.120.317134, indexed in Pubmed: 32302265.

21. Li J, Wang X, Chen J, et al. Association of Renin-Angiotensin System Inhibitors With Severity or Risk of Death in Patients With Hypertension Hospitalized for Coronavirus Disease 2019 (COVID-19) Infection in Wuhan, China. JAMA Cardiol. 2020 [Epub ahead of print], doi: 10.1001/jamacardio.2020.1624, indexed in Pubmed: 32324209.

22. Choi H, Koo HJ, Seok H, et al. ARB/ACEI use and severe COVID-19: a nationwide case-control study. MedRxiv. 2020, doi: 10.1 101/2020.06.12.20129916.

23. Hu J, Zhang X, Zhang X, et al. COVID-19 patients with hypertension have more severity condition, and ACEI/ARB treatment have no infulence on the clinical severity and outcome. J Infect. 2020 [Epub ahead of print], doi: 10.1016/j.jinf.2020.05.056, indexed in Pubmed: 32474032.

24. Zhou X, Zhu J, Xu T. Clinical characteristics of coronavirus disease 2019 (COVID-19) patients with hypertension on reninangiotensin system inhibitors. Clin Exp Hypertens. 2020; 42(7): 656-660, doi: 10.1080/10641963.2020.1764018, indexed in Pubmed: 32404011 .

25. Huang Z, Cao J, Yao Y, et al. The effect of RAS blockers on the clinical characteristics of COVID-19 patients with hypertension. Ann Transl Med. 2020; 8(7): 430, doi: 10.21037/atm.2020.03.229, indexed in Pubmed: 32395474.

26. De Spiegeleer A, Bronselaer A, Teo JT, et al. The Effects of ARBs, ACEis, and Statins on Clinical Outcomes of COVID-19 Infection Among Nursing Home Residents. J Am Med Dir Assoc. 2020; 21(7): 909-914.e2, doi: 10.1016/j.jamda.2020.06.018, indexed in Pubmed: 32674818.

27. Nicastri E, Petrosillo N, Ascoli Bartoli T, et al. National Institute for the Infectious Diseases "L. Spallanzani", IRCCS. Recommendations for COVID-19 clinical management. Infect Dis Rep. 2020; 12(1): 8543, doi: 10.4081/idr.2020.8543, indexed in Pubmed: 32218915.

28. Ip A, Parikh K, Parrillo J, et al. Hypertension and Renin-AngiotensinAldosterone System Inhibitors in Patients with Covid-19. MedRxiv. 2020, doi: 10.1101/2020.04.24.20077388.

29. Khera R, Clark C, Lu Y, et al. Association of Angiotensin-Converting Enzyme Inhibitors and Angiotensin Receptor Blockers with the Risk of Hospitalization and Death in Hypertensive Patients with Coronavirus Disease-19. medRxiv. 2020, doi: 10.1101/2020.05. 17.20104943, indexed in Pubmed: 32511481.

30. Peng YD, Meng K, Guan HQ, et al. [Clinical characteristics and outcomes of 112 cardiovascular disease patients infected by 2019$\mathrm{nCoV}$ ]. Zhonghua Xin Xue Guan Bing Za Zhi. 2020; 48(6): 450-455, doi: 10.3760/cma.j.cn112148-20200220-00105, indexed in Pubmed: 32120458. 
31. Reynolds HR, Adhikari S, Pulgarin C, et al. Renin-Angiotensin-Aldosterone System Inhibitors and Risk of Covid-19. N Engl J Med. 2020; 382(25): 2441-2448, doi: 10.1056/NEJMoa2008975, indexed in Pubmed: 32356628.

32. Richardson S, Hirsch JS, Narasimhan M, et al. and the Northwell COVID-19 Research Consortium. Presenting Characteristics, Comorbidities, and Outcomes Among 5700 Patients Hospitalized With COVID-19 in the New York City Area. JAMA. 2020 [Epub ahead of print], doi: 10.1001/jama.2020.6775, indexed in Pubmed: 32320003.

33. Chaudhri I, Koraishy F, Bolotova O, et al. Outcomes Associated with the Use of Renin-Angiotensin-Aldosterone System Blockade in Hospitalized Patients with SARS-CoV-2 Infection. Kidney360. 2020; 1(8): 801-809, doi: 10.34067/kid.0003792020.

34. Dublin S, Walker RL, Floyd JS, et al. Renin-angiotensin-aldosterone system inhibitors and COVID-19 infection or hospitalization: a cohort study. medRxiv. 2020, doi: 10.1101/2020.07.06.201203 86, indexed in Pubmed: 32676610.

35. Felice C, Nardin C, Di Tanna GL, et al. Use of RAAS inhibitors and risk of clinical deterioration in COVID-19: results from an Italian cohort of 133 hypertensives. Am J Hypertens. 2020 [Epub ahead of print], doi: 10.1093/ajh/hpaa096, indexed in Pubmed: 32511678.

36. Lam KW, Chow KW, Vo J, et al. Continued in-hospital ACE inhibitor and ARB Use in hypertensive COVID-19 patients is associated with positive clinical outcomes. J Infect Dis. 2020 [Epub ahead of print], doi: 10.1093/infdis/jiaa447, indexed in Pubmed: 32702098.

37. Şenkal N, Meral R, Medetalibeyoğlu A, et al. Association between chronic ACE inhibitor exposure and decreased odds of severe disease in patients with COVID-19. Anatol J Cardiol. 2020; 24(1): 21-29, doi: 10.14744/AnatolJCardiol.2020.57431, indexed in Pubmed: 32628137.

38. Meng J, Xiao G, Zhang J, et al. Renin-angiotensin system inhibitors improve the clinical outcomes of COVID-19 patients with hypertension. Emerg Microbes Infect. 2020; 9(1): 757-760, doi: 10.10 80/22221751.2020.1746200, indexed in Pubmed: 32228222.

39. Bean DM, Kraljevic Z, Searle T, et al. Angiotensin-converting enzyme inhibitors and angiotensin II receptor blockers are not associated with severe COVID-19 infection in a multi-site UK acute hospital trust. Eur J Heart Fail. 2020; 22(6): 967-974, doi: 10.1002/ejhf.1924, indexed in Pubmed: 32485082.

40. Yang G, Tan Z, Zhou L, et al. Angiotensin II Receptor Blockers and Angiotensin-Converting Enzyme Inhibitors Usage is Associ- ated with Improved Inflammatory Status and Clinical Outcomes in COVID-19 Patients With Hypertension. medRxiv. 2020, doi: 10. 1101/2020.03.31.20038935.

41. Feng Y, Ling Y, Bai T, et al. COVID-19 with Different Severities: A Multicenter Study of Clinical Features. Am J Respir Crit Care Med. 2020; 201(11): 1380-1388, doi: 10.1164/rccm.202002-0445OC, indexed in Pubmed: 32275452.

42. Feng Z, Li J, Yao S, et al. The Use of Adjuvant Therapy in Preventing Progression to Severe Pneumonia in Patients with Coronavirus Disease 2019: A Multicenter Data Analysis. medRxiv. 2020, doi: 1 0.1101/2020.04.08.20057539.

43. Rentsch CT, Kidwai-Khan F, Tate JP, et al. Covid-19 Testing, Hospital Admission, and Intensive Care Among 2,026,227 United States Veterans Aged 54-75 Years. medRxiv. 2020, doi: 10.1101/2020.0 4.09.20059964, indexed in Pubmed: 32511595.

44. Zeng Z, Sha T, Zhang Y, et al. Hypertension in patients hospitalized with COVID-19 in Wuhan, China: A single-center retrospective observational study. medRxiv. 2020, doi: 10.1101/2020.04.06. 20054825.

45. Sunden-Cullberg J. Chronic Use of Angiotensin-Converting Enzyme Inhibitors and Angiotensin II Receptor Blockers Is High Among Intensive Care Unit Patients With Non-COVID-19 Sepsis but Carries a Moderately Increased Risk of Death. Hypertension. 2020; 75(6): e15-e16, doi: 10.1161/HYPERTENSIONAHA.120.15178, indexed in Pubmed: 32275190.

46. Shinohara Y, Origasa H. Post-stroke pneumonia prevention by angiotensin-converting enzyme inhibitors: results of a metaanalysis of five studies in Asians. Adv Ther. 2012; 29(10): 900-912, doi: 10.1007/s12325-012-0049-1, indexed in Pubmed: 22983755.

47. Sriram K, Insel PA. Risks of ACE Inhibitor and ARB Usage in COVID-19: Evaluating the Evidence. Clin Pharmacol Ther. 2020; 108(2): 236-241, doi: 10.1002/cpt.1863, indexed in Pubmed: 32320478.

48. Gurwitz D. Angiotensin receptor blockers as tentative SARS-CoV-2 therapeutics. Drug Dev Res. 2020; 81(5): 537-540, doi: 10.1002/ ddr.21656, indexed in Pubmed: 32129518.

49. Liu Y, Yang Y, Zhang C, et al. Clinical and biochemical indexes from 2019-nCoV infected patients linked to viral loads and lung injury. Sci China Life Sci. 2020; 63(3): 364-374, doi: 10.1007/s11427020-1643-8, indexed in Pubmed: 32048163.

50. Aronson JK, Ferner RE. Drugs and the renin-angiotensin system in covid-19. BMJ. 2020; 369: m1313, doi: 10.1136/bmj.m1313, indexed in Pubmed: 32241880. 